\title{
Conocimiento y actitudes de los padres en relación a la vacunación de sus hijos
}

\author{
Liliana Véliz, Cecilia Campos y Paula Vega
}

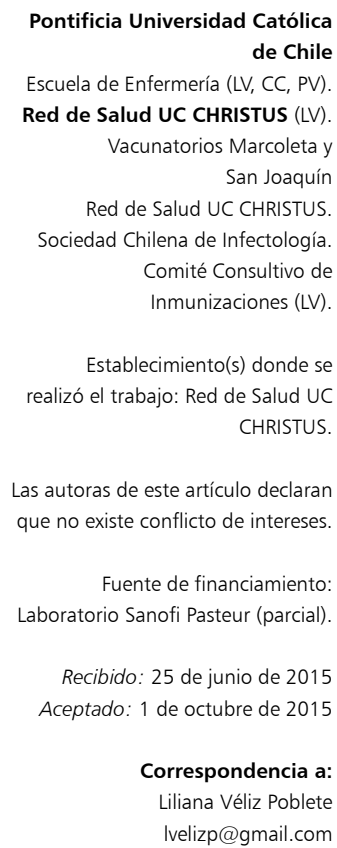

Pontificia Universidad Católica de Chile Escuela de Enfermería (LV, CC, PV) Red de Salud UC CHRISTUS (LV) Vacunatorios Marcoleta y San Joaquín Red de Salud UC CHRISTUS. Sociedad Chilena de Infectología. Comité Consultivo de Inmunizaciones (LV)

Establecimiento(s) donde se realizó el trabajo: Red de Salud UC CHRISTUS.

Las autoras de este artículo declaran que no existe conflicto de intereses.

Fuente de financiamiento: Laboratorio Sanofi Pasteur (parcial).

Recibido: 25 de junio de 2015 Aceptado: 1 de octubre de 2015

Correspondencia a: Liliana Véliz Poblete Ivelizp@gmail.com

\section{Introducción}

$\mathrm{P}$ ara enfermería el estudio de los conocimientos y actitudes permite valorar las capacidades de los usuarios y en función de ello, diseñar las intervenciones educativas necesarias y pertinentes para ayudarles a tomar decisiones informadas, reforzar sus capacidades de auto-cuidado y estimularlos a participar de mejor forma en cuidados de su salud o de la de los suyos ${ }^{1}$.

La vacunación es una acción sanitaria que ha permitido disminuir la mortalidad de los niños y erradicar del orbe enfermedades como viruela o en el continente americano la poliomielitis. Esta medida se considera altamente rentable para la promoción de la salud y la riqueza de las naciones. A mediados del siglo pasado, el esfuerzo de los equipos de salud para convencer a los padres de la importancia de esta medida sanitaria y lograr que la aceptaran, fue de gran magnitud. Más adelante, gracias al progreso del nivel educativo de la población y al avance de los programas de salud materno-infantil, se ha llegado a porcentajes altos de cobertura.

Sin embargo, esto hoy parece estar en riesgo. En Chile la cobertura de vacunación en la población infantil, según cifras del MINSAL año 2010, era sobre $90 \%$ para la mayoría de las vacunas indicadas durante los dos primeros años de vida. No ocurría lo mismo con las vacunas antidifteria, coqueluche y tétanos (DPT) indicada entonces a los 4 años de edad, cuya cobertura era de 78,8\%; la vacuna tres vírica (SRP) a aplicar cuando los menores cursaban el primer año de enseñanza básica y la vacuna toxoides diftérico/tetánico que correspondía cuando cursaban el segundo año de enseñanza básica, cuyas coberturas eran de 83,8 y $80,6 \%$, respectivamente ${ }^{2}$.

Los datos disponibles en el sistema de monitoreo de vacunas de la Organización Mundial de la Salud dan cuenta que todas las vacunas han disminuido su cobertura en comparación con el año $2007^{3,4}$.

Con preocupación, en los últimos tiempos se ha observado en determinados grupos de padres, cierta resistencia a que sus hijos sean inmunizados; ello como resultado de las campañas de los grupos anti-vacunas y de la información no científica ni validada a la que se tiene acceso actualmente a través de Internet y redes sociales. En este medio se pueden encontrar más de un centenar de estudios publicados en revistas científicas que reportan efectos negativos de las vacunas, categorizados por diferentes condiciones de salud ${ }^{5}$. Últimamente en Chile, se ha sumado a ello, la discusión surgida respecto a la reciente aprobación de la ley que prohíbe el uso de vacunas que contengan timerosal, que actualmente tiene veto presiden- 
cial $^{6}$. Esta ley busca prohibir el uso de timerosal en las vacunas administradas a niños bajo ocho años de edad, por la dudosa posibilidad de asociación con el autismo o enfermedades del neuro-desarrollo. Si bien hoy día existe suficiente evidencia científica que desmiente la relación entre autismo y otras enfermedades con aplicación de vacunas $^{7-9}$, un número no despreciable de padres se niega a vacunar a sus hijos. Los grupos anti-vacunas no sólo ponen en riesgo a sus propios hijos, sino que también a la población general, al afectarse la inmunidad de rebaño. Por ello, se hace necesario reconocer la inquietud actual existente en nuestro país y en el mundo en relación a las vacunas, con el fin de dar información clara y fundamentada a la población y así ayudar a los padres a tomar decisiones bien informadas en esta $\operatorname{area}^{10}$. Muchos padres vacunan a sus hijos sin tener un conocimiento de la protección que las vacunas otorgan, las edades y dosis en que se deben administrar y las enfermedades que evitan ${ }^{11}$. En forma especial llama la atención lo observado en los vacunatorios de la Red de Salud UC CHRISTUS, donde el nivel educacional de los padres que asisten a vacunar a sus hijos corresponde mayoritariamente a enseñanza media completa y superior; muchos de ellos no saben qué vacunas le corresponde a sus hijos y menos aún la edad y el número de dosis que le deben colocar para lograr la protección efectiva. Sin un adecuado conocimiento, ni una actitud favorable relacionada con la vacunación de sus hijos, el cuidado de ellos está en riesgo y se pone en peligro el éxito del Programa Nacional de Inmunizaciones (PNI) de Chile ${ }^{12}$. Desde antes del nacimiento, los padres son los principales responsables del cuidado de la salud de sus hijos, tomar las decisiones y medidas para proteger su vida y favorecer su crecimiento y desarrollo ${ }^{1,13}$.

Con el propósito de contribuir a la adherencia al PNI en Chile, la presente investigación tuvo como objetivo describir y analizar el conocimiento y las actitudes de padres de niños recién nacidos (RN) y lactantes menores y mayores, frente a la aplicación de las vacunas que conforman el PNI.

Se espera que los resultados entreguen información relevante que permita el desarrollo de programas educativos destinados a reforzar la importancia de la vacunación y el papel protagónico de los padres como responsables del cuidado de sus hijos ${ }^{14,15}$. Junto a la acogida y orientación del equipo de salud a los padres vacilantes ${ }^{16}$.

\section{Material y Método}

Estudio exploratorio, descriptivo y mixto (cuantitativo y cualitativo), realizado entre los meses de marzo y septiembre del año 2014, en una muestra por conveniencia, de 102 padres, cuyos RN eran sus primeros hijos (grupo 1) y de 101 niños de dos y más meses de edad, que asistieron a dos vacunatorios de la Red de Salud UC CHRISTUS, para la administración de las vacunas correspondientes a su edad (grupo 2). Previa obtención de consentimiento informado, se aplicó como instrumento de medición un cuestionario, elaborado en dos modalidades correspondientes a cada grupo: "Encuesta de Vacunas para Padres de RN" (Anexo 1) y "Encuesta de Vacunas para Padres en Vacunatorio" (Anexo 2).

La Encuesta de Vacunas para Padres de RN contiene siete preguntas cerradas y una abierta. Cada pregunta cerrada con tres alternativas de respuesta: sí, no, no sé. Se aplicó a los padres de los niños en la maternidad o en la primera consulta de RN. Ellos fueron contactados en el puerperio, previo al alta por las matronas del servicio o al momento de asistir a la consulta EPAS (Educación para el Autocuidado de la Salud) de RN, por las enfermeras a cargo de esas consultas.

La Encuesta de Vacunas para Padres en Vacunatorio contiene seis preguntas cerradas y dos abiertas y se aplicó a los padres de niños que acudieron a los vacunatorios. Ellos fueron contactados por los técnicos de enfermería o enfermeras de ambos vacunatorios, al momento de acudir a vacunar a sus hijos.

Las encuestas fueron elaboradas por las autoras de este estudio, tomando como referencia un cuestionario que se aplicó en España entre los años 1993 y $2003^{17}$. Previo a su aplicación se sometió a la validación de contenido por un grupo de tres expertos, enfermeras especialistas en cuidados infantiles y con experiencia clínica y docente en pediatría e inmunizaciones (Anexo 3).

Para las preguntas cerradas, el análisis de datos fue cuantitativo, descriptivo, en tablas de frecuencia. Para las preguntas abiertas se utilizó el diseño cualitativo de Análisis de Contenido, según Krippendorff ${ }^{18}$ con el objeto de identificar las categorías emergentes del discurso escrito. Este autor, define este diseño como una "técnica de investigación destinada a formular inferencias reproductibles, válidas y aplicables al contexto”, a partir de ciertos datos o contenidos latentes, los que dan respuesta a las interacciones simbólicas expresadas en el discurso del sujeto. Para el desarrollo de este análisis, las tres autoras analizaron las narrativas de los encuestados, para posteriormente realizar una triangulación entre dichos análisis y definir las categorías centrales y sus dimensiones.

El protocolo de este estudio fue aprobado por la comisión de ética clínica de la Escuela de Medicina de la Pontificia Universidad Católica de Chile (CECMedUC).

Para su ejecución se contó con una ayuda financiera parcial del laboratorio Sanofi Pasteur.

\section{Resultados}

\section{Aspectos generales}

El estudio se realizó en una muestra por conveniencia de dos grupos de padres: 102 de niños RN y 101 de 
niños de entre 2 y más meses de edad, a los que durante los meses de marzo a septiembre del año 2014 se invitó a participar; firmaron un consentimiento informado y respondieron la encuesta correspondiente a cada grupo. Por lo general fue la madre quien respondió la encuesta, la mayoría sin problemas, en un tiempo promedio de $7 \mathrm{~min}$.

\section{Tabla 1. Encuesta para padres de recién nacidos}

Frecuencia de respuestas ( $\mathrm{n}: 102)$ Sí No No sé n (\%) n (\%) n (\%)

¿Piensa que las vacunas sirven para curar enfermedades? 48 (47) 46 (45) 9 (9) ¿Piensa que las vacunas sirven para prevenir enfermedades? ¿Cree que las vacunas hacen daño al organismo? $\begin{array}{lllll}96 & (94) \quad 5 & (4,9) \quad 2 & (1,9)\end{array}$ ¿Piensa que las vacunas provocan la enfermedad más suave? ¿Cree que las vacunas son seguras? ¿Conoce las vacunas que debiera colocarse su hijo? $10 \quad(9,8) \quad 67 \quad(65,6) \quad 26 \quad(25,4)$ $49 \quad(48) \quad 35 \quad(34,3) \quad 19 \quad(18,6)$ $59 \quad(57,8) \quad 18 \quad(17,6) \quad 26 \quad(25,4)$ ¿Piensa vacunar a su hijo? $29 \quad(28,4) \quad 56 \quad(54,9) \quad 18 \quad(17,6)$ $93(91,1) \quad 4 \quad(3,9) \quad 6 \quad(5,8)$

Tabla 2. Encuesta para padres en el vacunatorio. Frecuencia de respuestas (n: 101)

\section{Pregunta}

¿Sabe qué vacuna le va a colocar a su hijo?

¿Sabe contra qué enfermedad/es protege la vacuna que le viene a colocar a su hijo?

¿Cree que la vacuna le provocará reacción con fiebre y malestar? ¿Usted usa paracetamol antes de vacunarlo para prevenir la fiebre?

¿Sabe qué vacuna le corresponde después de ésta? (1 ${ }^{\text {a }}$ fase) ¿Sabe qué vacuna le corresponde después de ésta? ( $2^{\mathrm{a}}$ fase) ¿Ha oído hablar del mercurio en las vacunas?

\begin{tabular}{clrcccc}
\multicolumn{2}{c}{ Sí } & \multicolumn{2}{c}{ No } & \multicolumn{2}{c}{ No Sé } \\
n & $(\%)$ & n & (\%) & n & $(\%)$ \\
94 & 93 & 5 & 5 & 2 & 2 \\
79 & 79,7 & 15 & 14,8 & 7 & 7
\end{tabular}

$\begin{array}{llllll}79 & 79,7 & 15 & 14,8 & 7 & 7\end{array}$

$\begin{array}{llllll}68 & 68,6 & 17 & 17 & 16 & 16\end{array}$

$\begin{array}{llllll}31 & 31,3 & 70 & 70,7 & 0 & 0\end{array}$

$\begin{array}{llllll}39 & 76 & 10 & 20 & 2 & 4\end{array}$

$\begin{array}{llllll}20 & 40 & 24 & 48 & 6 & 12\end{array}$

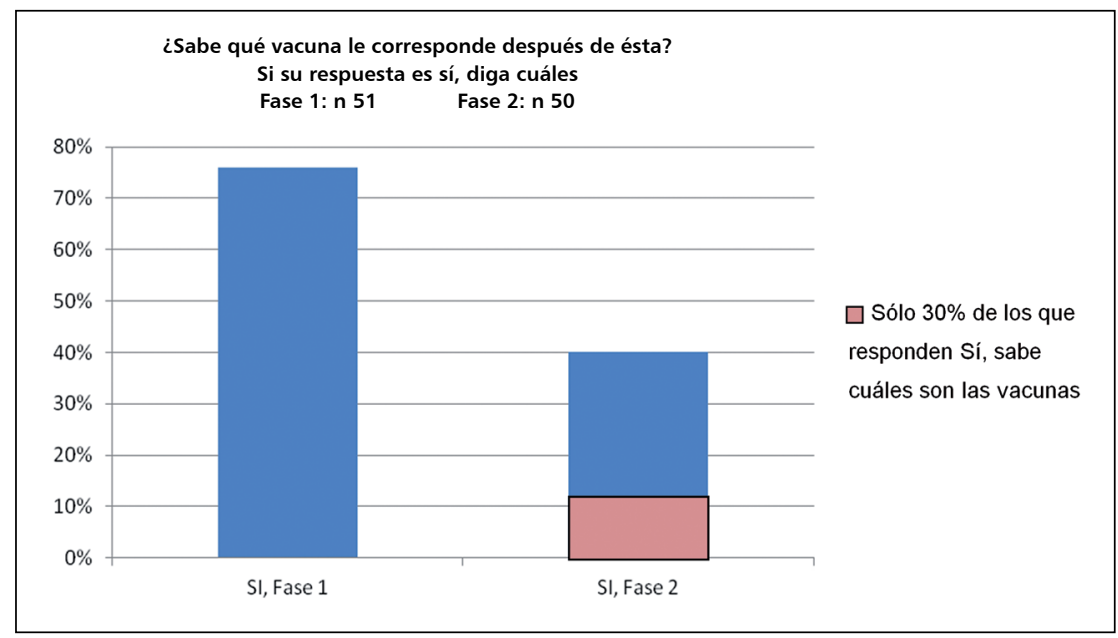

Figura 1.
El rango de edad de los padres fue de 16 a 58 años promedio de 29,5 años en las madres y 32,2 años en los padres. La edad de los niños, para el grupo 1 fue de 1 a 58 días y para el grupo 2, de 4 a 24 meses. El 85,2\% de los niños vive con ambos padres y $97 \%$ de los encuestados declara usar en forma habitual Internet.

\section{Análisis cuantitativo de preguntas cerradas}

Conocimiento de los padres de los $R N$ (grupo 1) sobre las vacunas del PNI vigente en Chile. Cerca de $100 \%$ piensa que las vacunas sirven para prevenir enfermedades y declaran estar dispuestos (intención de actuar) a vacunar a sus hijos (Tabla 1). Independientemente de este conocimiento y actitud, catalogado como muy bueno, el conocimiento respecto a las vacunas que le deberían colocar a sus hijos, no lo es; sólo $28,4 \%$ (pregunta 6) de los encuestados declara saber cuáles son estas vacunas.

En relación a la creencia respecto a si las vacunas pueden causar algún daño, $65,6 \%$ de los padres opinan que no lo causan, $9,8 \%$, piensa que sí pueden causar daño y $25,4 \%$ declara no saber. Con respecto a mitos, $47 \%$ de estos padres, creen que las vacunas sirven para curar enfermedades.

Conocimiento que tienen los padres de niños que ya han sido inmunizados (Grupo 2) sobre las vacunas del PNI vigente en Chile. El 93\% de ellos dice saber qué vacuna le corresponde colocar a su hijo (pregunta 1) (Tabla 2). Un análisis preliminar de resultados realizados en los 51 primeros casos, mostró que $76 \%$ respondió afirmativamente la pregunta “ ¿Sabe qué vacuna le corresponde después de ésta?" (pregunta 5). Sin embargo, dado que este resultado no es congruente con lo observado en la práctica, en la encuesta de los 50 padres restantes se incorporó la pregunta "Si su respuesta es Sí, diga cuál" (Anexo 4) y este resultado mostró que sólo 40\% contesta que sí sabe, pero de ese porcentaje, sólo 30\% nombró en forma correcta las vacunas que le corresponden (Figura 1).

Respecto al uso de paracetamol previo a la administración de vacunas (tema controversial en el ámbito médico, según lo observado en la práctica clínica) $70,7 \%$ de los padres dice no usarlo, aunque del total de los encuestados, $68,6 \%$ cree que la vacuna le provocará fiebre y malestar al niño.

Los resultados de la encuesta permiten deducir que los padres de estos niños entre dos y más meses de edad tienen, en general, alguna idea de las vacunas que su hijo debe recibir durante la infancia, pero no tienen el conocimiento preciso del calendario vigente, ni del nombre de las vacunas. Desde la perspectiva del auto-cuidado de la salud, estos padres, agentes del cuidado de sus hijos, podrían no dar un adecuado cumplimiento al calendario de inmunizaciones, lo que constituye un riesgo para la salud de esos niños. 


\section{Análisis cualitativo de preguntas abiertas}

El análisis de contenido según Krippendorff, que se realizó a los comentarios de los padres, develó que existen diferentes opiniones en relación a la vacunación de sus hijos.

En el caso de los padres de los RN (grupo 1), éstos consideran que la vacunación es una medida de prevención favorable para la salud de sus hijos. Sin embargo, algunos sostienen que la información existente no les permite tomar una decisión clara sobre la aplicabilidad de las vacunas y que incluso sospechan que hay intereses económicos tras éstas.

Para una mayor compresión de estos aspectos, a continuación se presentan las categorías identificadas con algunas narrativas que permiten confirmar lo analizado:

\section{Ser una medida de prevención favorable para la salud sus hijos}

Los padres de $\mathrm{RN}$ consideran que la vacunación es una medida de protección hacia sus hijos, al prevenir algunas enfermedades y favorecer la generación de anticuerpos necesarios contra éstas. A su vez, desean vacunar a sus hijos porque consideran que son una medida beneficiosa para ellos, y por lo mismo, ellos estiman que la vacunación en Chile es segura y que está científicamente comprobada su efectividad.

"Las vacunas ayudan al bebé para prevenir muchas enfermedades, lo que recomiendo $100 \%$ y me quedo más tranquila de que mi hijo va a estar libre de enfermedades con las vacunas suministradas".

"Al igual que con muchos tratamientos y medicamentos, me imagino que algunos podrian generar efectos colaterales no deseados. Sin embargo, y considerando el beneficio que otorgan en la prevención y control de enfermedades, prefiero utilizarlas, especialmente considerando que los daños de que se habla aún no son certeros ni comprobados totalmente".

"Un gran avance en cuanto a tecnología y medicina, que debiese estar al alcance de todos".

\section{La información existente no les permite tomar una decisión sobre la aplicabilidad}

Para algunos de los padres encuestados, existe aún cierto desconocimiento de la efectividad de estas vacunas, lo que les quita seguridad frente a considerarlo como una medida de protección para su hijo. Para ellos esto se debe a la existencia de mucha información errada en los medios masivos y a la poca información al interior de las instituciones de salud.

"Son una forma de prevenir enfermedades, pero se debería dar más información sobre ellas tanto positiva como negativa", "No tengo la certeza de su efectividad".

"Pienso que quizás haya vacunas que no son necesarias. Además hay mucha información contradictoria, algunos especialistas afirman que es bueno y otros que no".

\section{Una medida que puede ser dañina para los hijos, por manipulación de la información}

Algunos padres de RN no tienen confianza en la vacunación dado que afirman que son necesarias, pero que están conscientes que pueden ser dañinas para sus hijos, dado los componentes que tienen. Además, refieren sentirse muchas veces obligados a colocarlas, tanto por ley como por la presión de los profesionales de la salud. Incluso, algunos de ellos muestran incredulidad frente a esta medida, dado que lo consideran parte de un negocio de los laboratorios que desarrollan las vacunas.

"Que es un mal necesario"

"Pienso que la vacunación debiera ser libre, los padres decidir si vacunar a su hijo o no, sin temor a ser maltratados psicológicamente por no querer vacunar a su hijo y a posterior (ante enfermedad) que el bebé sea atendido sin problemas. Chile es uno de los países que más vacunas administran a niños menores de 8 años inclusive inyectando el virus al bebé por enfermedades que se encuentran sin brotes de hace años. Muchos sabemos que las vacunas, además, es el negocio de las industrias farmacéuticas que nada le conviene que seamos personas sanas. Así mismo, si todos supiéramos el compuesto de las vacunas (tejidos orgánicos de animales, virus y el famoso timerosal) muchos dudarian de sugerir la vacuna y otros, de ponerla", "Son ley y hay que ponerlas si o si aunque uno como padre no quiera (no me agrada)".

En el caso de los padres del grupo 2, que ya han vacunado a sus hijos, varios de ellos consideran que las vacunas son una medida favorable. Opinan, sin embargo, que la información existente no les permite estar seguros frente a los reales beneficios de éstas, sintiéndose algunos incluso obligados a hacerlo. Por lo tanto, lo consideran un mal necesario.

Narrativas que permiten confirmar lo analizado:

\section{Una medida favorable para la salud de su hijo/a}

La mayoría de los padres encuestados consideran que las vacunas son una medida de protección para prevenir enfermedades en sus hijos, por lo que son necesarias y beneficiosas para mantener la salud en ellos, lo cual además está apoyado en la creencia que son seguras y científicamente confiables.

"Se han creado tantas enfermedades que prefiero vacunar a mi hijo para prevenir cualquier enfermedad. Yo como madre crecí con las mismas vacunas, ¿por qué no vacunar a mi hijo si no hay nada comprobado en contra su uso?"

"Mi madre, cuando era pequeña, me las colocó y fueron efectivas y por el momento con mi hija también".

"Son formas de prevenir algo más grave que uno no sabe si sucederá y no me quiero arrepentir". 


\section{Falta información clara sobre las vacunas}

Algunos padres reconocen que existe desconocimiento en cuanto a la efectividad de las vacunas y la existencia de efectos secundarios indeseados, lo que genera en ellos algo de desconfianza.

"Se requiere mayor información".

"Me parece que han ayudado a mejorar la calidad de la salud infantil; sin embargo, frente a las polémicas suscitadas se podría informar en mayor medida a la opinión pública". "No he encontrado información concluyente al respecto, pero la vacuna hexavalente le provocó menos malestar".

\section{Es considerado un mal necesario}

Varios de los padres encuestados, opinan que la forma de administrar las vacunas, genera dolor en los niños, lo cual es indeseable y lo perciben como una medida impuesta.

"Que podrían buscar la forma de que sean menos invasoras".

"La vía de administración es poco amigable para niños y padres”.

"No me gustan, lo hago por obligación".

El análisis que se realizó a partir de la opinión que tenían los padres del grupo 2 respecto al uso del mercurio en las vacunas, mostró que ellos sí han escuchado hablar de este compuesto ( 75 padres de los 101).

El análisis de las narrativas según Krippendorff, mostró que existen tres tipos de opinión frente a este tema. Por una parte, un grupo de padres confía en su uso y considera que no es riesgoso. Otros, manifestaron que falta información, lo que les genera preocupación y un tercer grupo, considera que el uso del mercurio es nocivo para sus hijos, pero se ven obligados a vacunarlos. Llama la atención, que dos de los padres manifestaron no interesarse en el tema.

\section{Narrativas que permiten confirmar lo identificado}

\section{Es un compuesto inofensivo para el organismo}

Algunos padres consideran que los estudios demuestran que es seguro y además confían en las indicaciones de su pediatra y en las autoridades de salud. A su vez, sostienen que las vacunas no les causan daño a sus hijos, $y$ muy por el contrario, les permiten prevenir enfermedades.

"Me he informado del tema y por ende la cantidad del derivado del mercurio es menor en las vacunas".

"Confio plenamente en las investigaciones que se realizan y en la experiencia con todas las personas han demostrado que no es perjudicial en las dosis que corresponden".

"Consulté con su Pediatra para ver otras opciones y me indicó que la cantidad de mercurio contenida en las vacunas era inofensiva y no había nada seguro respecto del antiguo".

\section{Falta información al respecto}

Otro grupo de padres consideran que no existen los estudios suficientes que sean concluyentes con respecto a la toxicidad del mercurio y su uso en vacunas.

"No hay información, sólo especulación".

"Me gustaría tener más información actualizada de los componentes de las vacunas".

"No tengo una respuesta clara ya que hay controversia respecto a las vacunas con mercurio y sus repercusiones en la salud".

\section{Es un compuesto dañino para el organismo}

Un tercer grupo de padres, decidieron optar por vacunar a sus hijos con vacunas que no contuvieran mercurio. Algunos de ellos tienen la opinión que el Ministerio de Salud (MINSAL) debiera asumir los costos de vacunas más seguras, y no ser ellos quienes las deban costear.

"Debería haber un medio menos riesgoso para mantener las vacunas".

"Yo por lo menos prefiero prevenir y optar por vacunas que no lo contengan, además hasta donde entiendo, no sólo tiene relación con el autismo, sino con otras situaciones".

"Debieran eliminarlo y el Ministerio asumir el costo".

\section{Discusión}

Tanto el grupo de los padres de RN como los padres con experiencias anteriores de vacunación de sus hijos, consideran que las vacunas son una medida de protección frente a ciertas infecciones. Sin embargo, ambos grupos perciben que falta información con respecto a los componentes, las complicaciones, efectos adversos y la efectividad que realmente tienen las vacunas. Estos resultados concuerdan con lo encontrado los estudios de Raithatha y cols. ${ }^{10}$, y Bernal y cols. ${ }^{19}$. Estudios anteriores señalan que es la falta de información oficial en las instituciones de salud o el exceso de información no oficial a través de internet, lo que ha llevado a algunos padres a dudar sobre la necesidad de vacunar y a otros a sentir que más que una necesidad, es una obligación vacunar a los hijos ${ }^{5}$. Aunque el nivel de conocimiento sobre las vacunas en este grupo es muy bueno y las actitudes son positivas, es urgente y necesario insistir en la transmisión de información a los padres ${ }^{16,19,20}$. Más aún, porque existe poca evidencia acerca de la percepción de riesgo de vacunación entre padres que vacunan a sus hijos vs los que no lo hacen ${ }^{10}$.

Muchos de los padres que han oído hablar del compuesto mercurio en las vacunas, manifiestan temor frente a su supuesta relación con la aparición del autismo, lo que coincide con lo presentado en el estudio de Rebolledo $\mathrm{A}^{11}$. La relación entre las vacunas y el autismo se sustenta en el artículo de Wakefield y cols., que fue publicado en la revista The Lancet en el año 1998. Este artículo 
fue retirado en 2010 debido a serios problemas éticos y metodológicos ${ }^{7,9}$. A pesar de ello, esta preocupación sigue presente en muchos padres y las declaraciones oficiales respecto a la seguridad de las vacunas que contienen timerosal parecen no convencer a los grupos que se resisten a la vacunación ${ }^{21}$. A raíz de que muchas personas están dejando de vacunar a sus hijos, A. Carroll, autor del mapa interactivo de "brotes prevenibles por vacunación" en todo el mundo (2008 a 2014), muestra el dato geográfico de los brotes de distintas enfermedades controladas mediante vacunas, junto con los brotes típicos endémicos de los diferentes continentes. Este autor considera que no vacunar no es una decisión personal sino de salud pública ${ }^{22}$.

\section{Conclusiones}

En general, los padres participantes en este estudio manifiestan estar dispuestos a vacunar a sus hijos, pero necesitan contar con un mayor y fundamentado conocimiento que les permita tomar decisiones informadas. A ello se suma la necesidad de llegar a los grupos que definitivamente no vacunarán a sus hijos; no sabemos los pensamientos y actitudes de esos padres. Es importante considerar que mientras las personas no tengan los conocimientos ni se les aclaren con evidencias sus dudas, quedan en riesgo los planes de vacunación. El rol de los comités científicos es fundamental en este aspecto, por lo que les corresponde mantenerse alertas y actualizados en revisiones y evidencias acerca de las vacunas y sus componentes. Los profesionales de la salud, en especial pediatras y enfermeras, deben tener un diálogo empático con las personas, especialmente con las "vacilantes", con respuestas claras, precisas y convincentes. Es urgente aclarar dudas en torno a la seguridad de las vacunas desde el MINSAL y a nivel local.
Por lo descrito anteriormente, se hace necesario hacer más estudios de este tipo y abarcar más sectores de la población.

Agradecimientos. Las autoras agradecen al personal de los vacunatorios Marcoleta y San Joaquín, como también a las matronas del puerperio del Hospital Clínico UC y a las enfermeras de Pediatría del CMSJ y CEM, por su valiosa colaboración.

\section{Resumen}

Introducción: La vacunación, acción sanitaria que ha permitido disminuir la mortalidad y erradicar muchas enfermedades, puede estar en riesgo como resultado de la desinformación de los padres y campañas del movimiento anti vacunas. Objetivo: Describir y analizar conocimiento y actitudes de padres de recién nacidos y niños de dos y más meses de edad, en relación a la aplicación de vacunas. Método: Estudio exploratorio, descriptivo, mediante la aplicación de un cuestionario a padres asistentes a la Red de Salud UC CHRISTUS. Resultados: El conocimiento y actitud de los padres de $\mathrm{RN}$ es catalogado como muy bueno. El 35,2\%, piensa que las vacunas pueden causar daño y declara no saber y tiene conocimientos errados. Señalan que la información existente no les permite tomar decisiones correctas y sospechan intereses económicos detrás de los programas de vacunación. Los padres de niños mayores, no tienen conocimiento preciso del calendario vigente, ni del nombre de las vacunas. Opinan que la información existente no les permite estar seguros de los reales beneficios, sintiéndose algunos, obligados a vacunar a sus hijos. Discusión: Es urgente educar y aclarar dudas en torno a la importancia y seguridad de las vacunas y realizar estudios nacionales.

Anexo 1. Encuesta de vacunas para padres de recién nacidos

Edad del niño

Edad de la madre ___ Edad del padre

El niño vive con: ambos padres

sólo con la madre

¿Ud. usa habitualmente Internet?

Sí

No

sólo con el padre

\begin{tabular}{|c|c|c|c|}
\hline Pregunta & Sí & No & No sé \\
\hline ¿Piensa que las vacunas sirven para curar enfermeda & & & \\
\hline ¿Piensa que las vacunas sirven para prevenir enferm & & & \\
\hline ¿Cree que las vacunas hacen daño al organismo? & & & \\
\hline ¿Piensa que las vacunas provocan la enfermedad má & & & \\
\hline ¿Cree que las vacunas son seguras? & & & \\
\hline ¿Conoce las vacunas que debiera colocarse su hijo? & & & \\
\hline ¿Piensa vacunar a su hijo? & & & \\
\hline
\end{tabular}

Por favor escriba en una frase lo que usted piensa en relación a la aplicación de las vacunas: 
Anexo 2. Encuesta de Vacunas para Padres en el Vacunatorio (Fase 1)

Edad del niño

Edad de la madre ___ Edad del padre

El niño vive con: ambos padres___ sólo con la madre ¿Ud. usa habitualmente Internet? Sí Sí _sólo con el padre

No

\begin{tabular}{|c|c|c|c|}
\hline Pregunta & Sí & No & No se \\
\hline ¿Sabe qué vacuna le va a colocar a su hijo? & & & \\
\hline ¿Sabe contra qué enfermedad/es protege la vacuna que le viene a colocar a su hijo? & & & \\
\hline ¿Cree que la vacuna le provocará reacción con fiebre y malestar? & & & \\
\hline ¿Usted usa paracetamol antes de vacunarlo para prevenir la fiebre? & & & \\
\hline ¿Sabe qué vacuna le corresponde después de ésta? & & & \\
\hline ¿Ha oído hablar del mercurio en las vacunas? & & & \\
\hline
\end{tabular}

Si su respuesta № 6 es Sí, ¿Cuál es su opinión?:

Por favor escriba en una frase lo que usted piensa en relación a la aplicación de las vacunas:

Muchas gracias

\section{Anexo 3. Expertos que validaron los cuestionarios}

1. María Paz del Río Cea: Enfermera titulada en la Pontificia Universidad Católica de Chile, con diploma académico en Enfermería del Niño (2005), Instructora Adjunta Escuela de Enfermería UC, experiencia en atención ambulatoria infantil desde 2010.

2. Ana Verdugo Cárdenas: Enfermera titulada en la Pontificia Universidad Católica de Chile, Jefa Complejo Ambulatorio Marcoleta, experiencia en atención ambulatoria infantil e inmunizaciones desde 1991.

3. Francisca Villa Meléndez: Enfermera titulada en Universidad de Chile, experiencia en salud infantil desde 2003 y enfermera clínica del Vacunatorio Marcoleta desde 2010.

Anexo 4. Encuesta de Vacunas Padres en el Vacunatorio (Fase 2)

Edad del niño

Edad madre _Edad padre

El niño vive con: ambos padres

sólo con la madre sólo con el padre

¿Ud. usa habitualmente Internet? Sí __ No

\begin{tabular}{|l|l|l|l|}
\hline Pregunta & Sí & No & No se \\
\hline ¿Sabe qué vacuna le va a colocar a su hijo? & & & \\
\hline ¿Sabe contra qué enfermedad/es protege la vacuna que le viene a colocar a su hijo? & & & \\
\hline ¿Sabe qué vacuna le corresponde después de ésta? & & & \\
\hline
\end{tabular}

Si su respuesta № 3 es Sí, diga cuál vacuna:

\begin{tabular}{|l|l|l|l|}
\hline Pregunta & Sí & No & No se \\
\hline ¿Cree que la vacuna le provocará reacción con fiebre y malestar? & & & \\
\hline ¿Usted usa paracetamol antes de vacunarlo para prevenir la fiebre? & & & \\
\hline ¿Ha oído hablar del mercurio en las vacunas? & & & \\
\hline
\end{tabular}

Si su respuesta № 6 es Sí, ¿Cuál es su opinión?:

Por favor escriba en una frase lo que usted piensa en relación a la aplicación de las vacunas:

Muchas gracias 


\section{Referencias bibliográficas}

1.- Campos C, Jaimovich S. Autocuidado de la salud y crecimiento y desarrollo del niño. En: Meneghello, J Pediatría práctica en diálogos. Buenos Aires; Editorial Médica Panamericana; 2001. p. 172-5.

2.- Departamento de Estadística e Información de Salud. Indicadores básicos de Salud [Internet] Chile: Ministerio de Salud; 2010 [Consultado el 4 de abril de 2014]. Disponible en: http://deis.minsal.cl/deis/indicadores/ INDICADORES 2011.pdf.

3.- World Health Organization. Vaccine Preventable Diseases Monitoring System [Internet]; 2015 [Consultado el 15 de abril de 2014]. Disponible en: http://apps.who.int/immunization monitoring/globalsummary/countries?countrycri teria $\% 5 \mathrm{Bcountry} \% 5 \mathrm{D} \% 5 \mathrm{~B} \% 5 \mathrm{D}=\mathrm{CHL} \&$ commit $=\mathrm{OK}$

4.- Crocco P. El Plan Nacional de Inmunizaciones de Chile: ¿es una prioridad? Medwave. [Revista en línea] 2012 [Consultado el 24 de mayo de 2014]; 12 (11). Disponible en: http://www. medwave.cl/link.cgi/Medwave/Revisiones/ Analisis/5557

5.- Disiciencia. 100 estudios compilados sobre los peligros de las vacunas. [Internet]; 2012. [Consultado el 10 de mayo de 2015]. Disponible en: http://disiciencia.wordpress. com/2012/04/28/100-estudios-compiladossobre-los-peligros-de-las-vacunas/.

6.- Proyecto de Ley. Elimina las vacunas multidosis con timerosal o compuestos organomercúricos. Boletín N $N^{\circ}$ 7036-11. Cámara de Diputados (Consultado el 17 de diciembre de 2013).

7.- Farrington C, Miller E, Taylor B. MMR and autism: further evidence against a causal association. Vaccine. Junio de 2001; 19: 3632-5.

8.- Mäkelä A, Pekka J, Peltola H. Neurologic disorders after Measles-Mumps-Rubella vaccination. Pediatrics 2002; 110 (5): 957-63.

9.- Flaherty D. The Vaccine-Autism connection: A public health crisis caused by unethical medical practices and fraudulent science. Ann Pharmacother 2011; 45: 1302-4.

10.- Raithatha N, Holland R, Gerrard S, Harvey I. A qualitative investigation of vaccine risk perception amongst parents who immunize their children: a matter of public health concern. J Public Health Med 2003; (2): 161-4. DOI: 10.1093/pubmed/fdg034.

11.- Rebolledo A. Paremos la locura anti-vacunación infantil [Internet]. 2013 [Consultado el 5 de agosto de 2014]. Disponible en: http://www. elquintopoder.cl/ciencia/paremos-la-locura-antivacunacion-infantil/.

12.- Gellin B G, Maibach E W, Marcuse E K. Do parents understand immunizations? A national telephone survey. Pediatric 2000; 106 (5): 1097-102.

13.- Wroe A L, Turner N, Salkovskis M. Understanding and predicting parental decisions about early childhood immunizations. Health Psychology 2004; 23 (1): 33-41.

14.- Leandro Liberato S V, Hernández Galindo M, Cebrián Gimeno C, Elizalde Pellice C, Orrico Marín M A, Abeti Sarasa M A, et al. Vacunación infantil: Cobertura, conocimientos y actitudes de la población. Estudio en un Área de Salud. An Esp Pediatr 1996; 44 (5): 464-8.

15.- Díez-Delgado J, Lorente M J, Librada P, González M, Cañabate F, López F. Percepción de la vacunación por parte de los padres. An Esp Pediatr 1996; 45: 129-32.

16.- Healy C M, Pickering L K. How to communicate with vaccine-hesitant parents. Pediatrics. [Revista en línea] 2011; 127, (Suppl 1), Mayo: 127-133. DOI:10.1542/ peds.2010-1722S
17.- Carrasco-Garrido P, Gil de Miguel A. Hernández Barrera V, Vázquez-Fernández del Pozo S, Jiménez-Trujillo I, Jiménez-García R. Conocimientos de los padres españoles sobre la vacunación de sus hijos durante la década 1993-2003. Datos por comunidades autónomas. Vacunas 2006; 07 (4): 144-150. Disponible en: http://www.elsevier.es/es-revista-vacunas-72sumario-vol-07-num-04-13004162.

18.- Krippendorff K. Content analysis: An introduction to its methodology. Thousands Oaks: Sage Publications. 2004.

19.- Bernal P J, Níguez J C, Navarro J A. Actitudes, conocimientos, creencias y grado de satisfacción de los padres, en relación con las vacunaciones de sus hijos en la región de Murcia. Vacunas 2001; 2 (4): 142-148. Disponible en: http:// www.sciencedirect.com/science/article/pii/ S1576988701702555

20.- Boscan M, Salinas B, Trestini M L, Tomat M. Actitud de las madres en el cumplimiento del calendario de vacunación de niños menores de 6 años. Salus [Revista en Internet] 2012; 16 (1): 33-41. Disponible en: http://www.scielo.org.ve/ scielo.php?script $=$ sci_arttext\&pid $=$ S131671382012000100006\&lng=es.

21.- Muñoz A, Abarca K, Jiménez J, Luchslnger V, O'Ryan M, Ripoll E, et al. Seguridad de las vacunas que contienen timerosal: Declaración del Comité Consultivo de Inmunizaciones (CCI) de la Sociedad Chilena de Infectología. Rev Chilena Infectol 2007; 24 (5): 372-6.

22.- Carroll A. Mapa interactivo de "brotes prevenibles por vacunación" en todo el mundo desde 2008 hasta 2014. En Maltais M. Vaccinating your kids: Is it up to you or up to the government? [Internet] 2014. [Consultado el 24 de abril de 2014]. Disponible en: http://www. latimes.com/nation/la-sh-childhood-vaccine20140122-story.html\#axzz2rLmyyLCQ 